\title{
The partial thromboplastin (cephalin) time test
}

\author{
L. POLLER AND JEAN M. THOMSON
}

From the National Thromboplastin Centre, and the Haematology Department, Withington Hospital, University Hospital of South Manchester

SYNOPSIS Nine partial thromboplastin (cephalin) reagents have been compared in a paralle $\vec{P}$ investigation of groups of patients on 'long-term' anticoagulants, a group with moderate haemo $\vec{\omega}$ philia, and patients on heparin infusion.

Results with the seven commercial reagents and a human cephalin extract have been correlate울 with those of a specially prepared and standardized reference preparation of human brain origin The comparison was similar in principle to that of the prothrombin time thromboplastin standard $\vec{\sim}$ ization using the British Comparative Thromboplastin (BCT).

Results, which for comparative purposes were expressed as ratio of patients' cephalin times to control cephalin times, varied greatly in all three groups. In the oral anticoagulant group some of the? commercial reagents were particularly insensitive to the 'intrinsic' clotting defect. The correlationbetween the 'standardized preparation' and the other reagents was not good and the use of a reference्ष cephalin material for quality control of cephalin time tests does not appear promising.

In moderate haemophilia the commercial reagents were either relatively poor at picking out the clotting defect compared with the 'standardized preparation' or gave such a bad endpoint that the results were not dependable. The poor endpoint also limited the dependability of the results of \&lko but the 'standardized preparation' and two of the commercial reagents in controlling hepa in administration.

In view of these standardization difficulties, which cannot apparently be resolved by the use reference material, there is need for bulk, routine supplies of a sensitive, standardized cephalin reagent giving good reproducible endpoints. The method for the provision of such material in recently introduced national supply scheme is described.

The partial thromboplastin (cephalin) time is employed as an overall measure of 'intrinsic' blood clotting. Its main applications are in the screening for hereditary and acquired 'intrinsic' clotting defects and in assessing the intrinsic defect during oral anticoagulant treatment. The test involves the recalcification of platelet-poor plasma in the presence of a crude phospholipid extract. The origins of the latter vary and different animal tissues are used in their preparation. In addition 'home-made' phospholipid extracts from human brain may be made (Hjort, Rapaport, and Owren, 1955) and used at individual hospitals.
The present report describes the results encoun tered in clinical practice when a variety of reagents currently available in Britain are used. An attemp? has been made to correlate these reagents with 'standardized cephalin preparation'. The comparison of the cephalin reagents has been on patients on. 'long-term' anticoagulants (nicoumalone therapy) a group with moderately severe haemophilia, and patients on heparin therapy.

On the basis of the findings a system for standard izing the cephalin time test has been introduced and involving the supply of a sensitive standardized: cephalin preparation.

\section{Part I An assessment of the need for standardization}

\section{Method of Study}

Nine reagents, used as partial thromboplastins in Received for publication 18 September 1972. the cephalin time test, have been studied. These comprised seven preparations produced by com mercial manufacturers, ie, Platelin, Thrombofax; Thrombofax Activated, Diagen Platelet Substitute 
Diascreen, Hoechst and Hyland, and two of our own extracts of human brain (Hjort et al, 1955). Both human brain reagents, designated $A$ and $B$, were made in lyophilized form at the National Thromboplastin Centre. Batch $\mathbf{A}$ was produced in the usual way according to the original technique described by Hjort et al. With batch B the technique was modified by the addition of strict methods of quality control introduced to produce a sensitive and reproducible partial thromboplastin reagent. This is described in Part II. Batch human A was used in the coumarin study only.

During the performance of the cephalin time test maximum effort was made to avoid contact activation as follows: clean non-traumatic venepuncture; the use of non-wettable syringes and siliconized containers; storage of specimens in melting ice; centrifugation at $4^{\circ} \mathrm{C}$; the performance of the tests in parallel by six technicians and the limitation of the number of patients or controls to six on each day of testing. Two of the technicians who performed tests with more than one reagent tested each plasma in turn with the two cephalins. These stringent precautions were additional to the manufacturers' instructions which otherwise were followed. Results were the means of two duplicate tests on each patient.

With the human cephalin preparations $\mathbf{A}$ and $\mathbf{B}$ a kaolin-activated partial thromboplastin time was used, modified by Thomson (1970), from the method of Egeberg (1961), using a 10-minute activation period.

A ratio method was used for the expression of results, namely,

$$
\frac{\text { patient's cephalin time (sec) }}{\text { mean normal control cephalin time }(\mathrm{sec})}
$$

This seemed more valid for assessing the comparative sensitivity of the different reagents than simply recording the results of the individual cephalin times. The same principle is used with the British Comparative Thromboplastin when calibrating Quick prothrombin test reagents by correlation of results on anticoagulated patients (Poller, 1970). The reliability of the ratio system depends on accurate normal values. Twenty fresh normal specimens, taken from healthy adults were therefore tested individually with each reagent to give a good normal mean. The ratios obtained in the coumarintreated group with each cephalin reagent were plotted against the 'standardized preparation' (human cephalin B). Results were plotted on arithmetic graph paper with the 'standardized' preparation (human B) on the ordinate, as in the prothrombin time thromboplastin correlation.
The first group under study were stabilized 'longterm' patients on oral anticoagulant treatment. These normally show a prolongation of the cephalin time test which reflects depression of factors IX and $X$. The reliability and sensitivity of the available cephalin reagents were thus compared with the 'standardized preparation'. In the first part of the study, cephalin times were performed with each partial thromboplastin time reagent, in duplicate on 26 specimens from patients treated on a 'long-term' basis with nicoumalone, Sinthrome. These patients were adequately anticoagulated having prothrombin ratios greater than 2.0 with the British Comparative Thromboplastin.

The commercial reagent Thrombofax Activated only became available towards the end of the study and had to be compared individually with the 'standardized preparation' (human cephalin B) on the 17 coumarin plasmas.

In order to characterize the sensitivity of the reagents to moderate to severe factor VIII deficiency, seven plasmas from haemophilic patients whose factor VIII lay between 1 and $10 \%$ of pooled normal were tested individually with each commercial cephalin reagent and the 'standardized preparation'. The results in this series were also expressed as a cephalin ratio.

A study was also initiated on patients on heparin treatment to assess the sensitivity to the presence of circulating anticoagulant and suitability of the commercial reagents and 'standardized preparation'. The patients were on continuous heparin administration by continuous drip and their plasma recalcification times were approximately twice the upper limit of normality.

\section{Results}

\section{ORAL ANTICOAGULANT TREATMENT}

The results with the nine partial thromboplastin reagents included in the trial are given in Table $I$. The figures represent the mean ratio of the 26 coumarin plasmas with the eight cephalin reagents in Table I. Table I also gives the mean cephalin time (sec) of the 20 normal control plasmas tested with each reagent. The least sensitive reagents gave ratios of $1 \cdot 1$ whereas the two most sensitive reagents gave mean ratios of 1.4 on the same group of coumarin plasmas. There was no correlation between the length of the mean normal control time and the sensitivity, ie, no increase in sensitivity to compensate for the increasing tedium of the test arising from the longer clotting times. The results for Thrombofax Activated are given in parallel with the 'standardized reagent'. Individual patient's blood 


\begin{tabular}{lll}
\hline & Mean Ratio & $\begin{array}{c}\text { Mean Control } \\
\text { Time }(\mathrm{sec})\end{array}$ \\
\hline $\begin{array}{l}\text { On 26 plasmas } \\
\text { Thrombofax }\end{array}$ & $1 \cdot 1$ & \\
Hoechst & $1 \cdot 1$ & $87 \cdot 2$ \\
Human cephalin A & $1 \cdot 2$ & $45 \cdot 8$ \\
Platelin & $1 \cdot 2$ & $41 \cdot 1$ \\
Diagen & $1 \cdot 2$ & $95 \cdot 5$ \\
Diascreen & $1 \cdot 3$ & $49 \cdot 4$ \\
Human cephalin B & $1 \cdot 4$ & $56 \cdot 0$ \\
Hyland & $1 \cdot 4$ & $43 \cdot 0$ \\
& & $41 \cdot 5$ \\
On 17 plasmas & & \\
Thrombofax activated & $1 \cdot 2$ & $36 \cdot 5$ \\
Human cephalin B & $1 \cdot 5$ & $43 \cdot 0$ \\
\hline
\end{tabular}

Table I Results on coumarin plasmas.

showed even greater variation than the mean values given in Table $I$ indicated. Examples are given in Table II.

The comparison of the ratios of the different reagents with the standardized preparation, using the method recommended for comparison of Quick test thromboplastins and the BCT proved unworkable. When the results were plotted, in most instances it was impossible to draw a 'best line' by visual assessment with any degree of confidence owing to the degree of scatter of results. When the 'best line' was calculated on the basis of the formula for the regression line the extent of the divergence between the visual line and the calculated line with all of the reagents is seen in the figure. Where the lines coincide a correct discernible correlation was detected by eye. The divergence between the two lines indicates the unreliability of the procedure. There were too many aberrant points in five of the eight correlations. Alderson, Poller, and Thomson (1970) showed that with the British system for anticoagulant control when more than two aberrant points were present out of twelve, the correlation with the BCT was not valid. With prothrombin time ratios an aberrant point was defined as a point more than 0.5 of a ratio from the 'best line' in the vertical plane. With cephalin times the ratios are smaller when patients are at therapeutic levels of anticoagulant treatment but even on the liberal 0.5 ratio definition there were far too many aberrant points. The degree of scatter of the points was probably due to varying sensitivities of the cephalin reagents to different intrinsic clotting factors and probably also reflects different degrees of contact activation with the various techniques and reagents. The scatter was far greater than previously encountered with the comparison of tissue thromboplastins used in the prothrombin time test either by ourselves or hospitals participating in the national collaborative trials (Poller and Thomson, 1969; Alderson et al, 1970). It is relevant to note that the best correlations were obtained with comparisons of the two different human brain preparations $A$ and B, and of human B with Diagen and Diascreen.

PATIENTS WITH MODERATE HAEMOPHILIA The mean results of the seven patients, whose factor VIII levels ranged from $1 \%$ to $10 \%$, are given in Table IIIa. The mean ratio of the seven patients with each cephalin reagent gives an indication of the sensitivity of the reagent to factor VIII depletion. Comment on the endpoint obtained in the test is also given. With Thrombofax, the mean value could not be calculated because the endpoint was not recordable in four of the seven patients. Table IIIb gives the results with the haemophilic with a $10 \%$ factoo VIII level. With the exception of Diascreen all the reagents showed reasonable prolongation, ie, were sensitive to the defect at this level. Only the stand? ardized preparation, Diagen, and Diascreen gave good, reproducible endpoints. The ill-defined endpoints and poor reproducibility with the other reagents undermines their clinical value. The standardized preparation was the only sensitive preparation with a good, reproducible endpoint.

\section{PATIENTS ON HEPARIN TREATMENT}

Results with the two heparinized patients are given in Table IV. The heparin group was limited to two patients because it was found that the endpoint of the test was poor with all the commercial reagents except Diagen, which was good and Diascreen moderately good, at therapeutic levels of continuous heparin infusion. The best endpoint was with the standardized preparation (human B).

\begin{tabular}{|c|c|c|c|c|c|c|c|c|}
\hline Patient No. & $\begin{array}{l}\text { Human Brain } \\
A\end{array}$ & $\begin{array}{l}\text { Human Brain } \\
\text { B Standardized } \\
\text { Preparation }\end{array}$ & Diagen & Diascreen & Platelin & Thrombofax & Hoechst & Hyland \\
\hline $\begin{array}{l}1 \\
2 \\
3 \\
4 \\
5\end{array}$ & $\begin{array}{l}1.2 \\
1.3 \\
1.4 \\
1.9 \\
1.3\end{array}$ & $\begin{array}{l}1.6 \\
1.6 \\
1.8 \\
2.1 \\
1.7\end{array}$ & $\begin{array}{l}1.2 \\
1.2 \\
1.4 \\
1.8 \\
1.3\end{array}$ & $\begin{array}{l}1.4 \\
1.4 \\
2.0 \\
1.7 \\
1.4\end{array}$ & $\begin{array}{l}1.6 \\
1 \cdot 1 \\
1.3 \\
1.6 \\
1.3\end{array}$ & $\begin{array}{l}1.3 \\
1.0 \\
1.3 \\
1.5 \\
1.2\end{array}$ & $\begin{array}{l}1 \cdot 1 \\
1 \cdot 3 \\
1 \cdot 5 \\
1.4 \\
1 \cdot 0\end{array}$ & $\begin{array}{l}1.5 \\
1.4 \\
1.4 \\
2.6 \\
1.5\end{array}$ \\
\hline
\end{tabular}

Table II Examples of different cephalin ratios on five individual patients on nicoumalone (Sinthrome) 

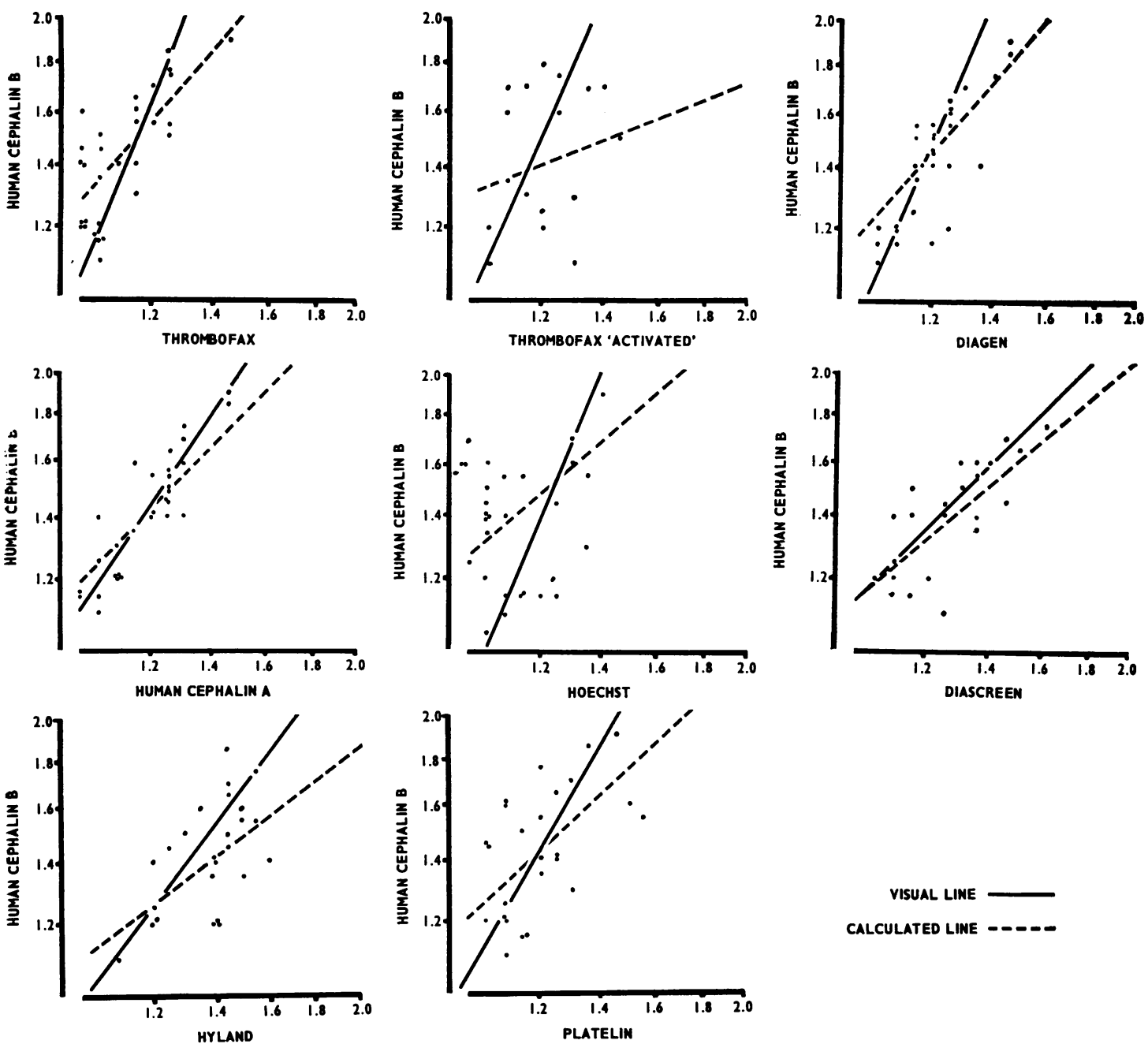

Fig. Comparison of cephalin ratios obtained with individual reagents and standardized preparation (human cephalin B) on patients receiving long-term anticoagulant treatment

\begin{tabular}{ll}
\hline Reagent & $\begin{array}{l}\text { Mean Cephalin } \\
\text { Ratio }\end{array}$ \\
\hline Human brain B (standardized) & $4 \cdot 6$ \\
Diascreen A & $2 \cdot 1$ \\
Platelin $^{1}$ & $2 \cdot 2$ \\
Diagen $^{1}$ & $2 \cdot 8$ \\
Hoechst $^{1}$ & $3 \cdot 2$ \\
Hyland $^{1}$ & $4 \cdot 1$ \\
Thrombofax A $^{1}$ & $5 \cdot 1$ \\
Thrombofax & Not recordable \\
\hline
\end{tabular}

Table III a Mean cephalin ratios with various reagents in patients with haemophilia

'Poor endpoints

\begin{tabular}{lll}
\hline & $\begin{array}{l}\text { Ratio(patientl } \\
\text { mean control) }\end{array}$ & Endpoint \\
\hline Human cephalin B & & \\
(standardized preparation) & 1.7 & Good \\
Thrombofax activated & 2.0 & Ill defined \\
Thrombofax & 1.8 & Almost unrecordable \\
Hyland & 1.8 & Poor \\
Hoechst & 1.4 & Poor \\
Platelin & 1.4 & Poor \\
Diagen & 1.2 & Good \\
Diascreen & 1.1 & Good \\
\hline
\end{tabular}

Table IIIb Results with haemophilic patient (10\% factor VIII level) 


\begin{tabular}{lll}
\hline & \multicolumn{2}{l}{ Cephalin Time Ratio } \\
\cline { 2 - 3 } & Patient 1 & Patient 2 \\
\hline Human cephalin B & & \\
'standardized preparation' & $2 \cdot 8$ & $1 \cdot 2$ \\
Hoechst & $2 \cdot 6$ & $1 \cdot 1$ \\
Diascreen & $2 \cdot 2$ & $1 \cdot 1$ \\
Thrombofax & 37 & $1 \cdot 2$ \\
Thrombofax A & 58 & $1 \cdot 4$ \\
Diagen & $4 \cdot 9$ & $1 \cdot 2$ \\
Platelin & $4 \cdot 0$ & $1 \cdot 2$ \\
Hyland & $2 \cdot 1$ & $1 \cdot 6$ \\
\hline
\end{tabular}

Table IV Results of patients on continuous heparin infusion

${ }^{1}$ Good endpoints

\section{QUALITY OF THE ENDPOINT}

The endpoint of the cephalin time test is important in ensuring the reliability of the result. A comparison has therefore been made of the quality of the endpoint with the various reagents in the different groups studied. Collective observations in all four groups are given in Table V. Only the human standardized preparation and Diagen gave good results with all four groups. Diascreen gave a good endpoint except for the patients on heparin where the endpoint was not clearly defined. Thrombofax reagent gave a poor endpoint throughout. The new activated Thrombofax reagent gave an improved endpoint with normals and the coumarin group whereas Hoechst and Hyland only gave good endpoints with the normal and coumarins. Platelin did not give good endpoints with any of the four groups, although not as bad as Thrombofax.

\section{Comments}

Patients on long-term anticoagulant treatment have a combined defect of factors II, VII, IX, and X and may have an induced anticoagulant, PIVKA (Hemker, Veltkamp, and Loeliger, 1968). Variation in sensitivity to specific clotting factors explains some of the differences between the mean ratios with the various cephalin reagents. Varying degrees of contact activation with the individual cephalin time

\begin{tabular}{lllll}
\hline & Normals & Coumarin & Haemophilia Heparin \\
\hline $\begin{array}{l}\text { Humancephalin B } \\
\text { 'standardized } \\
\text { preparation' }\end{array}$ & & & & \\
$\begin{array}{l}\text { Diagen } \\
\text { Diascreen }\end{array}$ & Good & Good & Good & Good \\
Platelin & Good & Good & Good & Good \\
Thrombofax & Moderate & Moderate & Poor & Moderate \\
Thrombofax A & Poor & Poor & Poor & Poor \\
Hoechst & Good & Good & Poor & Poor \\
Hyland & Good & Good & Poor & Poor \\
\hline
\end{tabular}

Table V Comparison of quality of endpoints in cephalin time tests techniques may be a contributing factor to the dis- $\frac{0}{5}$ crepant results. These uncontrolled variables in a $\stackrel{\text { ㅇ }}{\circ}$ group of patients might explain the enormous $\Rightarrow$ scatter of results when an attempt was made to $\stackrel{0}{\rightarrow}$ correlate the ratios on individual patients with the respective reagents (see figure). Only the three $\frac{\bar{F}}{\bar{\rho}}$ cephalin preparations-human A, Diagen, and $\frac{\bar{\sigma}}{\sigma}$ Diascreen - gave a correlation with the standardized $\stackrel{\Phi}{\varrho}$ preparation which was acceptable. In the prothrombin time test good correlations are normally ob- $\vec{\circ}$ tained between the Quick test reference reagent (British Comparative Thromboplastin) and other $\vec{\omega}$ Quick test reagents on coumarin blood samples. The cephalin time correlations are therefore disappointing.

The wide scatter in results indicates that there is i considerable variation in the ability of these cephalin $\vec{\sim}$ reagents to measure the coumarin-induced 'intrinsic' clotting defect. Obviously the larger the ratio with a $\vec{D}$ given cephalin and plasma, the greater the sen- $\infty$ sitivity and the more useful the reagent. Some of the reagents barely detect any change from normal at $\overrightarrow{ }$ therapeutic levels of oral anticoagulant dosage and $\nabla_{\mathbb{D}}$ are unsuitable for use in this context. It might be $\overparen{\mathbb{D}}$ possible to define a minimum sensitivity for such 3 preparations. It is apparent that even with animal $\underline{\Phi}$ tissue reasonable sensitivity to the coumarin defegt $\vec{\varphi}$ can be achieved and good quality endpoints obtained.

The impracticability of the correlation betwee the standardized cephalin material and the other partial thromboplastin reagents has important implications. It will clearly be impossible to standardize cephalin time results of coumarin-treated patients at different centres by the use of a cephalin $\overrightarrow{\vec{O}}$ reference preparation. If uniform results are to be 3 achieved the same reagent for use with a uniform technique will have to be supplied for routine work? to the hospitals.

The value of cephalin time tests as an adjunct to prothrombin times for controlling anticoagulant therapy is well established. We ourselves employ the test in parallel with the Quick test in anticoagulant control of our hospital patients and whenever any of our outpatients on anticoagulants require surgery or dental extraction. The test is also advisable if any $\frac{7}{0}$ patient experiences unexpected bleeding or further thrombosis occurs on anticoagulant treatment. In $N$ these circumstances to proceed without using a method sensitive to factor IX would be unwise. The $\mathrm{N}$ test has also been of value in the authors' experience $\omega$ in deciding whether to give vitamin $K_{1}$ in patients with excessively prolonged prothrombin times due to anticoagulant overdosage. Indeed Eastham (1968) has recommended the cephalin time as being more ? valuable than the Quick test in the regulation of long-term anticoagulant administration. 
The value of the cephalin time in the diagnosis of haemophilia has been a matter for dispute. Sawers and Hirsh (1970) state: 'The activated partial thromboplastin time test typically gives a prolonged clotting time in all grades of haemophilia except when the patient has recently received transfusion with fresh blood, or with poorly collected blood samples'. Most workers would accept the view that the partial thromboplastin time would probably give an abnormal result if the factor VIII was below $20 \%$ (Ingram, 1969).

In the present investigations the variable sensitivity of the commercial reagents to moderate haemophilia is seen and indicates the great variation in sensitivity of these preparations to low factor VIII levels. The mean cephalin ratios for the seven haemophilics varied from $2 \cdot 1$ to $5 \cdot 1$ (see Table III).
The prolongation of some of the tests was due to the indeterminate nature of the endpoint and not only to sensitivity to factor VIII levels. Only three reagents gave good endpoints, these were: the standardized material (human B), Diagen, and Diascreen, although the two commercial ones were relatively insensitive.

The cephalin time is also used for the clinical regulation of heparin dosage at some centres. The relative value of the different reagents in assessing the response is governed by two processes-the sensitivity to the anticoagulant effect of heparin and the quality of the endpoint. The results varied considerably (Table IV). Only the human standardized preparation, and possibly Diagen and Diascreen could be recommended for heparin control as the endpoint was too poorly defined with the others.

\section{Part II The preparation of a 'standardized cephalin' extract}

No system of quality control has hitherto been recommended for cephalin reagents and there has been little appreciation of the effect of variation of reagents on the sensitivity of the tests.

The first step in the production of a standardized cephalin preparation was to produce a crude phospholipid suspension which showed good sensitivity to the intrinsic clotting defect induced by oral anticoagulants. By modification of and experimentation with the Hjort method (1955) for the production of cephalin from human brain, more sensitive preparations were made. Initially our preparations were of the order of sensitivity of our human brain $A$ and varied considerably between batches.

The first problem therefore was to develop the maximum sensitivity to the intrinsic clotting defect. Many modifications of the human brain cephalin preparation were compared using results of stabilized patients on oral anticoagulant therapy. The aim was to develop the maximum sensitivity combined with reasonable prolongation of the normal value. It was also considered preferable to activate the plasma, as the unactivated cephalin method, although a good test, is more subject to variability in technique of blood collection and storage in vitro than the activated method. A 10-minute kaolin activation was chosen. The kaolin activation time with the different techniques varies from a minute or so up to 20 minutes. Even 10 minutes does not give absolutely complete activation and Hardisty and Hutton (1965) recommended 20 minutes. A 20-minute incubation time makes the test very lengthy, and, as activation is almost complete at 10 minutes, this time was preferred.

A large batch of human brain cephalin B has been prepared in lyophilized form. It is stored at $-70^{\circ} \mathrm{C}$, and degradation studies carried out at $50^{\circ} \mathrm{C}, 37^{\circ} \mathrm{C}$, room temperature, and $-20^{\circ} \mathrm{C}$ indicate that it should be stable at low temperature for a long time. Subsequent working batches of cephalin reagent have been made to conform with the standardized preparation, in a similar way to the method of preparation of the British Comparative Thromboplastin. Working batches of cephalin reagent have been offered to hospitals in the national system for anticoagulant control for clinical assessment. Over 200 hospitals have requested and received supplies of working cephalin reagent. If the preparation and cephalin time method recommended prove acceptable this could provide a basis for a national system for testing and reporting.

We are indebted to the technical staff of the above departments for assistance in the exercise.

The work was carried out whilst in receipt of a long-term grant from the Medical Research Council.

\section{References}

Alderson, M. R., Poller, L., and Thomson, Jean M. (1970). Validity of the British System for anticoagulant control using the national reagent. J. clin. Path., 23, 281-284.

Eastham, R. D. (1968). Improved control of long-term anticoagulant therapy. Brit. med.J., 2, 337-340.

Egeberg, O. (1961). Assay of antihaemophilic A, B and C factors by one-stage cephalin systems. Scand. J. clin. Lab. Invest., 13, 140-152.

Hardisty, R. M., and Hutton, R. A. (1965). The Kaolin clotting time of platelet rich plasma-a test of platelet factor 3 availability. Brit. J. Haemat., 11, 258-268.

Hemker, H. C., Veltkamp, J. J., and Loeliger, E. A. (1968). Kinetic aspects of the interaction of blood clotting enzymes: demonstration of an inhibitor of prothrombin conversion in Vitamin K deficiency. Thrombos. Diathes. haemorrh. (Stuttg.), 19, 346-363. 
Hjort, P., Rapaport, S. I., and Owren, P. A. (1955). A simple, specific one-stage prothrombin assay using Russell's viper venom in cephalin suspension. J. Lab. clin. Med., 46, 89-97.

Ingram, G. I. C. (1969). Laboratory identification of clotting defects. In Recent Advances in Blood Coagulation, edited by L. Poller, pp. 345-348. Churchill, London.

Poller, L. (1970). The British Comparative Thromboplastin: the use of the national thromboplastin reagent for uniformity of laboratory control of anticoagulants and expression of results.
L. Poller and Jean M. Thomson

Broadsheet 71, Association of Clinical Pathologists.

Poller, L., and Thomson, Jean M. (1969). The interpretation of 을 prothrombin results: a national survey. Brit. J. Haemat., 16, 31-37.

Sawers, R., and Hirsh, J. (1970). In Clinical Haematology in Medical Practice, 3rd ed., edited by G. C. de Gruchy, pp. 664-665. Blackwell, Oxford.

Thomson, Jean M. (1970). In A Practical Guide to Blood Coagulation and Haemostasis, pp. 175-176. Churchill, London. 